\title{
25 Wellen des Sozio-oekonomischen Panels (SOEP): Gewinn für interdisziplinäre Forschung
}

von Gisela Trommsdorff*

Auf ein Vierteljahrhundert eigenen Bestehens zurückzublicken, ist für eine Längsschnittsstudie wie das SOEP schon darum ein Grund zum Feiern, weil sicher ist, dass es nicht beim Rückblick bleiben wird, sondern dass neue Schritte in eine vielversprechende Zukunft anspruchsvoller und innovativer wissenschaftlicher Arbeit anstehen. Auf 25 Jahre wissenschaftliche Aktivitäten zurückzublicken ist für eine Institution - und das ist das SOEP inzwischen geworden - aber auch ein Anlass zu sichten, von welchen Aufgaben und Ressourcen einmal ausgegangen wurde, welche Entwicklungsschritte mit welchen Ergebnissen erfolgt sind und welche Ziele heute bestehen.

Das SOEP stellt seit 1984, also seit knapp 25 Jahren, in jährlichen Erhebungen repräsentative Datensätze (und daher mit dem Erhebungsjahr 2008 bereits 25 Wellen für die alten Bundesländer) zur Verfügung. Das SOEP wurde um Stichproben aus den neuen Ländern (seit 1989; 20. Welle im Jahre 2009) sowie immer wieder um Spezialstichproben (u.a. Migranten, Wohlhabende) erweitert.

\section{Institutionelle Veränderungen des SOEP}

Die Idee für ein „Sozio-oekonomisches Haushalts-Panel“ entstand Anfang der 1980er Jahre. Das SOEP wurde 1982 erstmals im Rahmen des Sfb 3 Frankfurt und Mannheim (Sonderforschungsbereich 3: Mikroanalytische Grundlagen der Gesellschaftspolitik), dessen Gründer der Ökonom Hans-Jürgen Krupp zusammen mit dem Soziologen Wolfgang Zapf war, durch die DFG mit finanzieller Unterstützung des BMFT gefördert. Die damaligen Gutachtergremien der DFG waren bereits interdisziplinär zusammengesetzt (u. a. gehörte auch der einflussreiche Entwicklungspsychologe und spätere Direktor des MPI München F. E. Weinert der Begehungsgruppe an) (vgl. die Übersicht bei Krupp 2007 sowie in diesem Heft).

Von 1982 bis 2002 wurde das SOEP als ein DFG-Projekt (mit entsprechenden Antragsund Berichtsverfahren) durchgeführt, mit erheblicher finanzieller Unterstützung des BMBFT bzw. später des BMBF und Überlassung von Räumen und Verwaltung, IT-Kapazität und anfänglich 3,5, später 2,5 Mitarbeiterstellen durch das DIW Berlin. 2003 wurde das SOEP als eigene Abteilung und Serviceeinrichtung innerhalb der Wissenschaftsgemein-

*Universität Konstanz, E-Mail: Gisela.Trommsdorff@uni-konstanz.de 
schaft Gottfried Leibniz (WGL) dem DIW angeschlossen. ${ }^{1}$ Nachdem Hans-Jürgen Krupp die Leitung des SOEP 1988 abgab (da er Senator für Finanzen der Freien und Hansestadt Hamburg wurde), leitet seit 1989 bis heute Gert G. Wagner das SOEP als Principal Investigator (vgl. hierzu Hanefeld und Schupp in diesem Heft).

Ein internationaler und interdisziplinär zusammengesetzter (wissenschaftlicher bzw. später Nutzer-)Beirat (seit 1993 unter meiner Mitwirkung und von 2004 bis 2007 unter meiner Leitung) begleitet die Arbeit des SOEP kontinuierlich. ${ }^{2}$ Die Beratungen und Evaluationen erfolgen u. a. hinsichtlich der Ziele und der dazu geeigneten Verfahren, der wissenschaftlichen Ergebnisse und der Vernetzung des SOEP mit anderen Institutionen.

Die verschiedenen einschneidenden organisatorischen Transformationen hat das SOEP trotz unübersehbarer Friktionen an verschiedenen Stellen nicht nur gut überstanden, es hat auch neue Herausforderungen vor allem in Bezug auf die interdisziplinäre Ausrichtung der eigenen und der angeregten Forschung aufgesucht und ist an den neuen Aufgaben gewachsen. Durch den Weitblick der Gründer des Sonderforschungsbereichs 3 und der Initiatoren des SOEP waren bereits Voraussetzungen dafür geschaffen, dass nicht nur ein Haushaltspanel eingerichtet wurde, um wirtschaftwissenschaftliche Fragen für Politikberatung zu beantworten. Diesen Spielraum hat das SOEP aufgrund seiner einmaligen und umfassenden Datenerhebung zur Einkommenslage von Privathaushalten im Quer- wie Längsschnitt genutzt, um auch darüber hinaus sozial- und verhaltenswissenschaftliche Forschung zu betreiben und anzuregen. Die Entstehung und der Entwicklungsverlauf des SOEP erscheinen heute eng verbunden mit der globalen Geschichte der empirischen sozial- und wirtschaftswissenschaftlichen Forschung, die zunehmend disziplinäre Grenzen zu überwinden versucht.

\section{Inhaltliche Weiterentwicklung des SOEP}

Im Laufe eines prospektiven Panels werden zum einen Änderungen und Anpassungen an besondere soziale, wirtschaftliche und politische Ereignisse erforderlich, wie z.B. durch den Beitritt der Neuen Bundesländer zur Bundesrepublik. Zum anderen werden aufgrund neuer theoretischer und methodischer Entwicklungen entsprechende Veränderungen in Fragestellungen und Methoden notwendig. Schließlich bringt es die jährliche Fortführung der Erhebung mit sich, dass die ursprünglichen Befragten älter werden und sich deren Entwicklungsaufgaben in Beruf und Familie verändern (wie durch Eltern- und Großelternschaft; Scheidung o.a.), und dass sie eines Tages durch Tod aus dem Panel ausscheiden. Diese längsschnittlichen Veränderungen erfordern eine ständige Überprüfung relevanter theoretischer Fragestellungen und Methoden sowie damit verbundene Anpassungen der Befragungsinhalte und Veränderung im Erhebungsdesign (Erweiterung um neue Teilstichproben wie die neuen Bundesländer, Migranten).

Welche Änderungen sowohl in der Erhebungsmethode als auch im Erhebungsprogramm des SOEP wissenschaftlich fruchtbar sein müssten, wird im Beirat unter Berücksichtigung der unterschiedlichen Präferenzen einzelner Disziplinen erörtert. Die methodische und

1 Die mit der neuen Institutionalisierung verbundenen Probleme der Governance, Unklarheiten hinsichtlich der Selbständigkeit des SOEP als Abteilung und der Rolle des Beirats des SOEP (dessen Aufgaben und Zuschnitt mehrmals gewechselt wurden) haben zeitraubende Konflikte geschaffen.

2 Eine Liste der SOEP-Beiratsmitglieder findet sich im Anhang. 
organisatorische Umsetzung der aus keineswegs immer einvernehmlichen Diskussionen hervorgehenden Empfehlungen des Beirates erfolgt dann jedoch unter Verantwortung der SOEP-Leitung. Hier besteht immer das Problem, die ,richtige“ Entscheidung zum richtigen Zeitpunkt zu treffen. Ein falsches Timing stellt ein beträchtliches Risiko dar.

Die richtige Entscheidung, die neuen Bundesländer bereits im Juni 1990 in die Erhebung mit einzubeziehen, ist einer Reihe von Faktoren zu verdanken, wie der Intuition der verantwortlichen Wissenschaftler, der Flexibilität der Feldorganisation von Infratest Sozialforschung (München) und nicht zuletzt der rechtzeitigen Bereitstellung finanzieller Ressourcen durch die Drittmittelgeber (im Falle der Stichprobe C des BMFT).

In ein Haushaltspanel, das so konzipiert ist, wie es das SOEP von Anfang an war, werden auch systematisch jüngere Kohorten einbezogen. Inzwischen ist es möglich, die Kinder und Enkel der bisher Befragten in die Erhebung mit aufzunehmen und damit Fragen zu Intergenerationenbeziehungen direkt vergleichend zu untersuchen und nicht aus der Retrospektive (Szydlik und Schupp 1998). Neuerdings wurden spezielle Erhebungsinstrumente für Kinder und Jugendliche eingeführt, weil inzwischen die achte Geburtskohorte des SOEP 17 Jahre alt wird (und damit zu eigenständigen Befragungspersonen). Zusätzlich zu den 2002 und 2005 erstmals gestellten Fragen (an die Eltern) in Bezug auf Neugeborene und Kleinkinder wird 2008 zum ersten Mal ein Fragebogen über Vorschulkinder verwendet, um kognitive und nicht-kognitive Kompetenzen zu erfassen. Da auch nicht-kognitive Kompetenzen wie Selbstregulation und Motivation von erheblicher Bedeutung für ökonomische Fragen (z.B. der Bildungs- und Berufsplanung oder Alterssicherung) sind, eröffnen sich neue Perspektiven für interdisziplinäre Forschungen. Inzwischen sind die Kohorten der älteren Bevölkerung im SOEP ausreichend vertreten, so dass für diese Gruppen entwicklungspsychologische Untersuchungen zu Stabilität und Veränderung von diversen Verhaltensmerkmalen über eine längere Lebensspanne möglich sind (vgl. Diener, Lucas und Scollon 2006). Darüber hinaus war aufgrund technischer Neuerungen die Erprobung und Anwendung neuer Erhebungsmethoden (von persönlichen Face-to-face-Interviews zu computergestützten Interviews) erforderlich. Damit wurden Grundlagen für empirisch abgesicherte Methodenentwicklungen gelegt, die wiederum exzellente Methodenkenntnisse und kontinuierliche Schulung voraussetzen.

Solche Neuerungen des SOEP in Bezug auf Design, inhaltliche und methodische Fragen erfordern erhebliche Kompetenzen aller am Survey beteiligten Wissenschaftler, neue Fragestellungen als wissenschaftlich relevant zu erkennen. Dies ist dem SOEP gelungen aufgrund einer sehr guten Einbindung in eine international ausgewiesene Scientific Community und einer hohen Flexibilität bei der Planung und Durchführung. Deswegen haben die für das SOEP Verantwortlichen auch die Chance ergriffen, die sozial- und verhaltenswissenschaftlichen Faktoren wirtschaftlich relevanten Handelns zu untersuchen und damit das Panel über ökonomische Fragen hinaus zunächst für soziologische und inzwischen u. a. auch für demografische, gesundheitspolitische, psychologische und verhaltensgenetische Fragen zu öffnen. Im Folgenden soll dies am Beispiel der Einführung psychologischer Konzepte und deren Messung illustriert werden.

Der Schritt zu den individuellen Grundlagen sozialen Handelns wurde durch das SOEP 1984 schon früh mit bis heute vorherrschenden Fragen nach Bedingungen von Lebenszufriedenheit über die Lebensspanne gelegt (vgl. Wagner, Frick und Schupp 2007). Damit entstanden Innovationen, die im Beirat (nicht nur anfangs) kritisch und kontrovers disku- 
tiert wurden. Die Forschung zu sozialen Indikatoren zeigte, dass nicht nur die üblichen objektiven Indikatoren wie Einkommen, sondern auch subjektive Indikatoren in das SOEP einbezogen werden sollten. Dies hat sich - ausweislich der vielen entsprechenden Publikationen - eindeutig bewährt und die Forschung deutlich bereichert. Der Boom der sogenannten „Happiness“-Forschung (,positive psychology“) hat sich in den letzten Jahren auch auf die wirtschaftswissenschaftliche und demografische Forschung zur Lebenszufriedenheit ausgewirkt.

Für Ökonomen ist die Lebenszufriedenheit u. a. im Zusammenhang mit Wohlstand und Arbeitsproduktivität von Interesse (Rätzel 2007). In der demografischen Forschung werden Zusammenhänge von Lebenszufriedenheit und veränderten Familienformen untersucht (Zimmermann und Easterlin 2006). In der Psychologie stellt sich vor allem die Frage, ob sich ökonomische Bedingungen wie Wohlstand auf eine erhöhte Lebenszufriedenheit auswirken oder ob Lebenszufriedenheit ein relativ stabiles Merkmal über die Lebenspanne ist, das von anderen als ökonomischen Faktoren beeinflusst wird. Zur Beantwortung dieser Frage sind die SOEP-Datensätze besonders geeignet, weil hier nicht nur querschnittliche Analysen zu Zusammenhängen, sondern auch im Längsschnitt Analysen über den Einfluss von Lebensereignissen auf die Lebenszufriedenheit möglich sind.

Aufgrund repräsentativer SOEP Daten belegen z. B. Rammstedt (2007) und Headey (2008) Zusammenhänge zwischen Persönlichkeitsmerkmalen (Big Five, Kontrollorientierung, Optimismus sowie Lebenszielen) einerseits und Lebenszufriedenheit andererseits. Damit stellt sich jedoch die nächste Frage, ob globale Persönlichkeitsmerkmale, Temperament und genetische Faktoren die Lebenszufriedenheit vorhersagen können oder ob andere Faktoren wie Sozialisationsbedingungen (in der Familie), Lebensereignisse und ökonomische Bedingungen eher relevant sind. Querschnittanalysen von Trzcinski und Holst (2006) belegen signifikante Zusammenhänge zwischen Wohlbefinden der Eltern (Mütter) und ihrer jugendlichen Kinder, aber nicht zwischen ökonomischen Bedingungen (Haushaltseinkommen) und Wohlbefinden der Jugendlichen.

Die Frage ist nach wie vor nicht beantwortet, ob Längsschnittanalysen relativ stabile (aber individuell verschiedene) Einstellungen über die Lebensspanne hinweg nachweisen, also ob davon auszugehen ist, dass individuelle Unterschiede darin bestehen, dass das Leben generell eher als positiv oder als negativ erlebt wird und zwar relativ unabhängig von ökonomischen Bedingungen.

Nun ist inzwischen auch Kritik an der bisherigen, schon „traditionell“" gewordenen Messung von Lebenszufriedenheit laut geworden. Die lange verwendeten, gut validierten Items zur Messung subjektiven Wohlbefindens erfassen eher kognitive und vernachlässigen emotionale Komponenten. Damit stellte sich eine erneute Herausforderung an das SOEP, zu prüfen, wie das theoretische Konstrukt der Lebenszufriedenheit weiter differenziert und methodisch valide erfasst werden kann. Diese Frage wurde auch im Beirat kontrovers diskutiert, weil für Wissenschaftler verschiedener Disziplinen nicht ohne weiteres einsichtig ist, dass auch Emotionen als ein relevantes Konstrukt im SOEP mit erfasst werden sollten. Zudem sind Emotionen auch in der Psychologie erst relativ spät entdeckt worden. Inzwischen werden sie bei Ökonomen im Zusammenhang mit Fragen der „,bounded rationality“ aber auch nicht mehr vollständig ignoriert. Auf Initiative des Ökonomie-Nobelpreisträ- 
gers Daniel Kahneman, der Psychologie-Professor an der Princeton University ist, ${ }^{3}$ wurde erstmals im Jahr 2007 eine Kurz-Skala zu „Affekten“ in das SOEP aufgenommen (vgl. Schimmack, Schupp und Wagner 2008).

Das SOEP hat schon früh, auch Dank der Weitsicht seiner Gründer, weitere psychologische Konzepte und Fragestellungen berücksichtigt, die in der psychologischen Forschung seit langem untersucht, aber bisher in der Wirtschafts- und Sozialwissenschaft kaum eine Rolle gespielt haben. Sie haben dann aber in den letzten Jahren trotz anfänglichen Widerstandes einzelner Kollegen durch Aufnahme in das SOEP zur Untersuchung wichtiger Forschungsfragen beigetragen.

Ebenfalls in der Erprobung sind (für psychologische und verhaltensökonomische Fragen relevante) experimentelle Methoden, u.a. auch zur Messung von Selbstregulation bei Kindern und Jugendlichen. So wichtig das hier zugrunde liegende Ziel ist, Einstellungsdaten durch Verhaltensbeobachtungen zu ergänzen, ist hier ein besonders ausgeprägtes methodisches Problembewusstsein erforderlich. Dazu können die gerade anlaufenden Validierungsstudien des SOEP einen wichtigen Beitrag leisten. Die meisten Vorversuche und Validierungsstudien wurden zudem nicht durch das normale Budget des SOEP finanziert; vielmehr wurden hierfür Drittmittel eingeworben. Die Notwendigkeit der Einwerbung von Drittmitteln garantiert, dass es nicht zu einer unkritischen Verwendung methodisch noch nicht final validierter Verfahren kommt.

Zusammenfassend lässt sich sagen, dass das SOEP mit der Einführung von Konzepten aus nicht sozialwissenschaftlichen Disziplinen, insbesondere in letzter Zeit aus der Psychologie, ganz wesentlich zu Befruchtungen zwischen den verschiedenen Forschungsgebieten beigetragen hat. Die damit einhergehende Methodenarbeit des SOEP hat dazu geführt, gut validierte Instrumente zur Untersuchung von Fragestellungen hervorzubringen, die für verschiedene Disziplinen von erheblicher Bedeutung sind. Das SOEP hat so eine anderweitig kaum realisierbare Weiterentwicklung von Methoden und darauf aufbauende interdisziplinäre Forschung angeregt.

Für die psychologische Forschung selbst sind unter anderem folgende Aspekte der SOEPDatensätze von besonderer Bedeutung. Die repräsentativen Datensätze des SOEP erlauben,

- theoretisch relevante Teilstichproben (Angehörige verschiedener Familienformen, Generationen, Bildungsgruppen u. a.) über die Zeit hinweg systematisch (auch im Vergleich zueinander) zu untersuchen,

- externe Faktoren (z. B. elterliche Erziehung, Bildung, Eintritt bestimmter Lebensereignisse, soziale Netze) und interne Faktoren (Persönlichkeitsmerkmale, Gesundheit) zu isolieren, welche die Entwicklungen über den Lebensverlauf (z. B. Gesundheits- und Risikoverhalten, Bildungs- und Berufskarrieren) beeinflussen,

3 Weitere Initiatoren waren Ed Diener (Psychologe, University of Illinois), Ruut Venhooven (Soziologe, University of Amsterdam), Bruce Headey (Soziologe, Melbourne Institute of Applied Economic and Social Research), Richard Lucas (Psychologe, Michigan State University) und Ulrich Schimmack (Psychologe, University of Toronto Mississauga). 
- Wechselwirkungen von Entwicklungsfaktoren im Zeitverlauf zu prüfen und somit dynamische Prozesse über die Lebensspanne zu modellieren.

So kann empfohlen werden, bei komplexen Fragestellungen zur Interaktion von Kontext und Individuum in der Lebensspanne vor Beginn einer eigenen Datenerhebung zu prüfen, welche Möglichkeiten das SOEP bietet. Ein Nachteil des SOEP für Psychologen ist allerdings, dass nur einige wenige psychologische Konzepte dort bisher Eingang gefunden haben. Mit guten Argumenten können jedoch, wie sich gezeigt hat, sinnvolle Ergänzungen für zukünftige Erhebungen vorgeschlagen werden, wenn sie auch für die anderen beteiligten sozialwissenschaftlichen Disziplinen relevant sind. Schon jetzt aber bietet das SOEP ganz sicher mehr aufschlussreiche Datensätze als dies bisher in der Psychologie bekannt ist.

Zusammenfassend lässt sich sagen, dass ein wichtiger Beitrag des SOEP für interdisziplinäre Forschung einerseits darin besteht, dass psychologische Konzepte, Theorien und Methoden in anderen Disziplinen genutzt werden, und dass andererseits eine fruchtbare Erweiterung psychologischer Forschung durch das SOEP und die am SOEP mitwirkenden Disziplinen ermöglicht wird. Das SOEP hat mit der Einführung von Konzepten aus nicht sozialwissenschaftlichen Disziplinen, insbesondere in letzter Zeit aus der Psychologie, ganz wesentlich zu Befruchtungen zwischen den verschiedenen Disziplinen beigetragen. Die damit einhergehende Methodenarbeit des SOEP hat dazu geführt, gut validierte Instrumente zur Untersuchung von Fragestellungen hervorzubringen, die für verschiedene Disziplinen von erheblicher Bedeutung sind. Das SOEP hat so eine anderweitig kaum realisierbare Weiterentwicklung von Methoden und darauf aufbauende interdisziplinäre Forschung angeregt.

\section{Gegenwart und Ausblick}

SOEP-Datensätze werden von Nutzern verschiedener Disziplinen für Longitudinalanalysen, für international vergleichende Studien und für Methodenanalysen der Umfrageforschung verwendet. Die sozial- und verhaltenswissenschaftliche Forschung profitiert in Deutschland und international von den Arbeiten des SOEP insbesondere in Bezug auf die empirisch fundierte Bearbeitung von Themen, die außerhalb der Möglichkeiten von Einzelwissenschaftlern oder auch größeren Institutionen sind.

Bezeichnend für die Arbeiten des SOEP sind die viel zitierten Publikationen sowohl der Mitarbeiter als auch der Nutzer, die sich international und interdisziplinär zusammensetzen und die grundlagenwissenschaftliche und anwendungsrelevante (u. a. gesundheits- und arbeitsmarktpolitische) Fragen behandeln. Hochrangige Beiträge von Wissenschaftlern verschiedener Länder zu aktuellen u. a. soziologischen, ökonomischen, demografischen, politikwissenschaftlichen und psychologischen Themen haben das Forschungspotential der SOEP-Datensätze für die Grundlagenforschung deutlich gemacht.

Wissenschaftliche Beiträge auf Grundlage von SOEP-Datensätzen werden inzwischen auch auf nicht-ökonomischen und nicht-soziologischen Kongressen vorgestellt. Ein Beispiel ist der 45. Kongress der Deutschen Gesellschaft für Psychologie in Nürnberg mit einer interdisziplinären Arbeitsgruppe zu SOEP-Studien, organisiert von SOEP-Mitarbeiter Markus M. Grabka, sowie der 29th International Congress for Psychology (ICP) in Berlin 
mit einem interdisziplinären und internationalen Symposium, organisiert von Trommsdorff und Lang (2008).

Das SOEP hat inzwischen auch Modellfunktion für andere wissenschaftlich fundierte Panels angenommen wie für das britische, australische und schweizerische Haushaltspanel sowie für die in Deutschland in Vorbereitung befindlichen Familien- und Bildungspanels.

In Großbritannien wird gerade eine massive Erweiterung der longitudinalen Haushalts-Erhebungen vorbereitet (UK HLS), um vor allem auch psychologische Fragen der Entwicklung über die Lebensspanne im Zusammenhang mit strukturellen Variablen der Veränderungen im Lebenslauf zu untersuchen. Bei einer ähnlichen Erweiterung würden für das SOEP ganz neue Möglichkeiten entstehen, die psychologische Lebensspannenforschung mit sozioökonomischen Fragen veränderter Kontexte im Lebenslauf zusammenzubringen - ein Anliegen, das Glen Elder (1974) und seine Kollegen bereits seit einigen Jahrzehnten erfolgreich mit amerikanischen Längsschnittdaten verfolgen. In der Psychologie entfaltet diese Idee durch Wiederentdeckung des ,ökologischen“ Kontextes für menschliches Fühlen, Denken und Handeln eine neue theoretische Dynamik. Die empirische Basis für diese Forschung können prospektive Panelstudien bilden, wie sie das SOEP darstellt. Wichtig dafür ist die Zusammenführung mit entsprechenden Datensätzen anderer Länder, um international vergleichende Studien durchzuführen und Fragen der Universalität und Kulturspezifität von sozioökonomischer und psychologischer Entwicklung zu bearbeiten. Insofern ist zu hoffen, dass das bislang von ökonomischen und gesundheitswissenschaftlichen Variablen dominierte Cross National Equivalent File (CNEF), das bislang Paneldaten für Australien, Deutschland, Großbritannien, Kanada, die Schweiz und die USA enthält (vgl. Frick et al. 2007 und in diesem Heft), auch um subjektive und verhaltenswissenschaftliche Variablen erweitert wird.

Die Frage, ob Grund besteht, das 25-jährige Jubiläum des SOEP zu feiern, erscheint als eine rhetorische Frage, wenn man die Vergangenheit und die Zukunftsperspektiven des SOEP betrachtet. Für das SOEP besteht ein besonderes Jubiläum, weil sich mit 25 Erhebungswellen Entwicklungsschritte ereignet haben, die heute zu einer ganz neuen Dimension von Forschungsaktivitäten führen. Die bisherige Arbeit des SOEP hat sich in vieler Hinsicht schon jetzt als gut dokumentierter Fortschritt erwiesen, mit vielfältigen Folgen für das SOEP selbst sowie für die Infrastruktureinrichtungen deutscher Sozialwissenschaften und für die internationale sozial- und verhaltenswissenschaftliche Forschung. Eher stellt sich daher die Frage, wie man ein solches Jubiläum denn konkret feiern kann und sollte.

Zum Ersten ist dieses Jubiläum eine hervorragende Gelegenheit, sowohl Fragen der optimalen Institutionalisierung des SOEP als auch der inhaltlichen Gestaltung des zukünftigen Forschungsprogramms weiter kompetent, einfallsreich und zukunftsorientiert zu diskutieren.

Zum Zweiten ist das Jubiläum eine sehr geeignete Gelegenheit, den vielen Personen, die seit der frühen Vorbereitung des SOEP aktiv an der Förderung und Entwicklung des SOEP mitgewirkt haben, einen besonderen Dank für ihren Weitblick und ihren Einsatz zum Ausdruck zu bringen. Das bedauerliche Schicksal des geplanten - und im Frühjahr 2008 gescheiterten - großen Schweizer entwicklungspsychologischen Kinder- und Jugendpanels (SESAM) belegt, wie schwierig die Einrichtung eines fundierten Panels ist, wenn die aktive Beteiligung der Bevölkerung in Frage steht. Das heißt auch, wie sehr die Existenz 
und der Erfolg eines Panels nicht nur abhängen von wissenschaftlicher, finanzieller und organisatorischer Förderung, sondern auch von der Bereitschaft der Bevölkerung, eine solche Einrichtung durch freiwillige Mitwirkung an den Befragungen zu unterstützen. Dies wird oft vergessen.

Anlässlich des Jubiläums des SOEP sollte ein besonderer Dank an die Befragten des SOEP gehen, die sich über all die Jahre hinweg an dem Panel beteiligt haben. Sie sollten erfahren, dass sich ihr Einsatz lohnt.

\section{Literaturverzeichnis}

Diener, Ed, Richard E. Lucas und Christie Napa Scollon (2006): Beyond the Hedonic Treadmill - Revising the Adaption Theory of Well-Being. American Psychologist, 61 (4), 305-314.

Elder, Glen H., Jr. (1974): Children of the Great Depression: Social change in life experience. Chicago, University of Chicago Press.

Frick, Joachim R., Stephen P. Jenkins, Dean R. Lillard, Oliver Lipps und Marc Wooden (2007): The Cross-National Equivalent File (CNEF) and its Member Country Household Panel Studies. Schmollers Jahrbuch, 127 (4), 627-654.

Headey, Bruce (2008): Life Goals Matter to Happiness: A Revision of Set-Point Theory. Social Indicators Research, 86 (2), 213-231.

Krupp, Hans-Jürgen (2007): Das Sozio-oekonomische Panel - Wie es dazu kam. In: Johannes Schwarze, Jutta Räbinger und Reinhold Thiede (Hrsg.): Arbeitsmarkt- und Sozialpolitikforschung im Wandel. Hamburg, Dr. Kovač, 15-39.

Rammstedt, Beatrice (2007): Who Worries and Who Is Happy? Explaining Individual Differences in Worries and Satisfaction by Personality. Personality and Individual Differences, 43 (6), 1627-1634.

Rätzel, Stefan (2007): Ökonomie und Glück - zurück zu den Wurzeln? Wirtschaftsdienst, 87 (5), 335-344.

Schimmack, Ulrich, Jürgen Schupp und Gert G. Wagner (2008): The Influence of Environment and Personality on the Affective and Cognitive Component of Subjective WellBeing. Social Indicator Research (im Druck).

Szydlik, Marc und Jürgen Schupp (1998): Stabilität und Wandel von Generationenbeziehungen. Zeitschrift für Soziologie, 27 (4), 297-315.

Trommsdorff, Gisela und Frieder R. Lang (2008): Psychological research with the German socio-economic panel. Symposium conducted at the XXIX International Congress of Psychology. Berlin, Juli 2008.

Trzcinski, Eileen und Elke Holst (2007): Initial Predictors of Life Satisfaction in Early Adulthood. Schmollers Jahrbuch, 127 (1), 95-104.

Wagner, Gert G., Joachim R. Frick und Jürgen Schupp (2007): The German Socio-Economic Panel Study (SOEP): Scope, Evolution and Enhancements. Schmollers Jahrbuch, 127 (1), 139-169.

Zimmermann, Anke C. und Richard A. Easterlin (2006): Happily Ever After? Cohabitation, Marriage, Divorce, and Happiness in Germany. Population and Development Review, 32 (3), 511-528. 